\title{
Response: Event-related brain dynamics - unifying brain electrophysiology
}

\section{Scott Makeig}

In large part, the study of brain electrophysiology now consists of nearly distinct fields: averaged event-related potentials (ERPs), the ongoing electroencephalogram (EEG), local field potentials (LFPs) and intracellular dynamics (most commonly, spike rate or timing) (Fig. 1). Of these, the two extreme subfields (ERPs and single-cell spike histograms) have been dominant, isolated from each other by differences in spatial scale, recording subjects and, in part, by modeling based on simple averaging methods. Our recent paper in Science [1] points out the conceptual impoverishment that results in the case of scalp ERPs, and demonstrates how more adequate analysis of single trials can find dynamic consistencies between features of EEG-trial averages (i.e. ERPs) and event-related changes in the ongoing EEG signals.

Meanwhile, other laboratories are exploring links between the timing of neuronal spikes and the dynamics of local-field potentials [2]. Connections between local field potentials and scalp EEG signals are still poorly understood and deserve further study, in which independent component analysis (ICA) $[1,3]$ might also prove useful.

Penny et al. [4] rightly place our recent paper on event-related EEG dynamics into the broader context of event-related brain dynamics, as studied in nonlinear dynamicsystems theory and at the level of groups of interconnected neurons. For neuroscience, the study of field dynamics has three

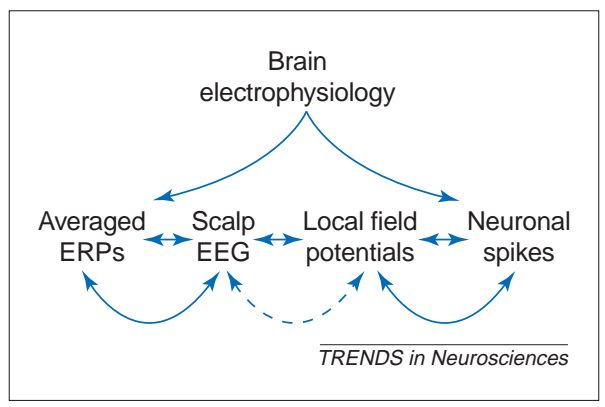

Fig. 1. Neuroscience has largely ignored relationships between four linking subfields of brain electrophysiology. Averaged event-related potentials (ERPs) (recorded from the human scalp) and single-neuron spike histograms (recorded from animals) have dominated, each largely isolated from the other by ignorance of their relationships to ongoing potentials recorded within the brain or on the scalp. This bifurcation has been nurtured by focus on the results of simple response-averaging methods (scalp ERPs, spike histograms). More adequate modeling methods are now demonstrating intimate relationships between all four electrophysiological domains during active cognitive processing.

advantages over single-cell spike train analysis. First, each recorded field signal indexes important (although still poorly understood) physiological processes within neuropile that are largely invisible in, but influence, spike recordings. Second, it is now possible to record fields

simultaneously at as many as hundreds of sites over most of the brain, to separate the resulting data into independent and/or transiently dependent signals, and to study the relationship of dynamic changes in these signals and their interrelations to behavior and experience. Third, recent results complement longstanding observations that distinct changes in the dynamics of brain fields accompany changes in attention [5], memory [6] and awareness [7], strongly suggesting that field dynamics play a role in top-down cognitive processing [8], of which the physiology is still largely mysterious.

\section{References}

1 Makeig, S. et al. (2002) Dynamic brain sources of visual evoked responses. Science 295, 690-694

2 Fries, P. et al. (2002) Oscillatory neuronal synchronization in primary visual cortex as a correlate of stimulus selection. Proc. Natl. Acad. Sci. U. S. A. 22, 3739-3754

3 Jung, T-P. et al. (2001). Imaging brain dynamics using independent component analysis. Proc. Inst. Electr. Electronic Eng. 89, 1107-1122

4 Penny, W. et al. (2002) brain dynamics. Trends Neurosci. 25, 387-389

5 Worden, M.S.et al. (2000) Anticipatory biasing of visuospatial attention indexed by retinotopically specific alpha-band electroencephalography increases over occipital cortex. J Neurosci. 20, RC63

6 Sarnthein, J.et al. (1998) Synchronization between prefrontal and posterior association cortex during human working memory. Proc. Natl. Acad. Sci. U. S. A. 7092-7096

7 Makeig, S. and Jung, T-P. (1996) Tonic, phasic, and transient EEG correlates of auditory awareness during drowsiness. Cogn. Brain Res. 4, 15-25

8 von Stein, A. et al. (2000) Top-down processing mediated by interareal synchronization. Proc. Natl. Acad. Sci. U. S. A. 27, 14748-14753

\section{Scott Makeig}

Swartz Center for Computational

Neuroscience, Institute for Neural

Computation, University of California

San Diego, La J olla, CA 92093-0961, USA.

e-mail: smakeig@ucsd.edu

\section{Cyclic amplification of protein misfolding: application to prion-related disorders and beyond}

\section{Claudio Soto, Gabriela P. Saborio and Laurence Anderes}

Diverse human disorders, including the majority of neurodegenerative diseases, are thought to arise from the misfolding and aggregation of protein. We have recently described a novel technology to amplify cyclically misfolded proteins in vitro. This procedure, named protein misfolding cyclic amplification (PMCA), is conceptually analogous to DNA amplification by PCR and has tremendous implications for research and diagnosis. The PMCA concept has been proved on the amplification of prions implicated in the pathogenesis of transmissible spongiform encephalopathies. In this article we describe the rational behind PMCA and some of the many potential applications of this novel technology. 